\title{
REGULARITY RESULTS FOR SEMILINEAR AND GEOMETRIC WAVE EQUATIONS
}

\author{
JALAL SHATAH \\ Courant Institute, 251 Mercer St., New York, N.Y. 10012, U.S.A. \\ E-mail: shatah@math13.cims.nyu.edu
}

1. Introduction. In this paper we will give an exposition of the ideas and techniques involved in proving well posedness and regularity of solutions to the Cauchy problem of semilinear wave equations. Our starting point is based on the treatment of the Cauchy problem

$$
\begin{aligned}
& u_{t t}-\Delta u+g(u)=0, \quad x \in \mathbb{R}^{3}, \\
& u(x, 0)=u_{0}(x), \quad u_{t}(x, 0)=u_{1}(x),
\end{aligned}
$$

by Jörgens [11] and Segal [19]. Their work laid the foundation for developing energy methods and $L^{p}$ estimates to prove existence of solutions to the above equation. The work of Strichartz [26] on estimates for the linear wave equation on $\mathbb{R}^{n}$ plays a central role in our exposition. These estimates were used effectively to prove existence and scattering of solutions to subcritical problems, as can be seen in the work of Brenner and von Wahl [2], Ginibre and Velo [6], Pecher [16], and Strauss [25] to mention a few. Moreover all of our regularity proofs for critical problems are based on energy estimates coupled with Strichartz estimate. In addition to these estimates the Morawetz identity also plays an important part in the regularity proof of critical problems, as was demonstrated by the work of Struwe [27], Grillakis [7], Kapitanski [12], and Shatah and Struwe [20, 21]. The proofs sketched in this exposition for global solvability of critical semilinear equations are essentially those presented in [20,21].

Another problem that we study in the manuscript is the Cauchy problem for wave maps. These are maps from Minkowski space into a complete Riemanian manifold $u$ : $\mathbb{R}^{n+1} \longrightarrow(N, g)$ that are solutions to the Cauchy problem

$$
\begin{aligned}
& D_{\alpha} \partial_{\alpha} u^{a}=\partial^{\alpha} \partial_{\alpha} u^{a}+\Gamma_{b c}^{a}(u) \partial_{\alpha} u^{b} \partial^{\alpha} u^{c}=0, \\
& u(0, x)=U_{0}(x), \quad \partial_{t} u(0, x)=U_{1}(x) .
\end{aligned}
$$

1991 Mathematics Subject Classification: 35L10, 35L15.

Research supported in part by the National Science Foundation grant DMS-9401558.

The paper is in final form and no version of it will be published elsewhere. 
Based on scaling the cut off space for local well posedness in $H^{\frac{n}{2}}\left(\mathbb{R}^{n}\right)$ (see Section 4.2). Up to this writing there is no proof that $H^{\frac{n}{2}}$ is the optimal space for local existence. For the general problem the best existence results in two space dimensions are given by Grillakis [9] and in three space dimensions by Klainerman and Machedon [13]. Both of these results require the data to be in the space $H^{\frac{n}{2}+\varepsilon}$ for arbitrarily small $\varepsilon$. The proof of this fact utilizes very different estimates based on null forms. These null forms estimates are better suited to handle derivative nonlinearities than the Strichartz estimates. Here we will give a simple proof based on energy estimates and the geometric nature of the equations that the problem is well posed in $H^{\frac{n+1}{2}}\left(\mathbb{R}^{n}\right)$.

Using energy estimates and Strichartz estimates we will show that for equivariant maps the problem is well posed in $H^{\frac{n}{2}}\left(\mathbb{R}^{n}\right)$ for $n \geq 2$. For $n=2$ we use a variant of the Morawetz identity to prove that with a certain condition on the metric the problem is globally well posed in $H^{1}\left(\mathbb{R}^{2}\right)$. This condition was given by Grillakis [8] and is an improvement over the convexity condition given by Tahvildar-Zadeh and the author in [22]. The proof that we will present here was given in [23].

For space dimensions $n \geq 3$ we will show that for some target manifolds $N$ there are smooth initial data such that the corresponding Cauchy problem develops a singularity in finite time. The conditions on $N$ in three space dimensions are geometric while for five space dimensions the conditions are analytic. In particular there are manifolds with negative sectional curvature such that the Cauchy problem develops singularities in finite time. These singular solutions lead to the existence of initial data in $H^{s}\left(\mathbb{R}^{n}\right) \forall s<\frac{n}{2}$ such that the Cauchy problem has more than one weak solution. Thus we prove that for equivariant maps, in space dimensions $n \geq 3, H^{\frac{n}{2}}\left(\mathbb{R}^{n}\right)$ is the optimal space for existence and uniqueness. These results were proved by Cazenave, Tahvildar-Zadeh and the author [3].

Notation. We use standard notations to denote function spaces. Besides the usual Lebesgue spaces $L^{p}$, Sobolev spaces $W^{s, p}$ and $H^{s}=W^{s, 2}$, we denote Besov spaces by $B_{q}^{s}$. A thorough treatment of these spaces can be found in Triebel [10] and Bergh and Löfström [1]. For a definition of the local version of the homogenous spaces

$$
\begin{aligned}
& \dot{H}^{s}(K)=\text { Homogenous Sobolev space, } \\
& \dot{B}_{q}^{s}(K)=\text { Homogenous Besov space, }
\end{aligned}
$$

the reader is referred to [20], page 508. For functions defined on space time, $L^{q}\left(L^{p}\right)$ denotes the space of functions that are $L^{p}$ in space and $L^{q}$ in time. The same notation is also used for other space time norms.

2. Estimates for the linear wave equation. In this section we will discuss energy and $L^{p}$ estimates for solutions of the linear wave equation. These estimates lead us to the Strichartz estimates which we will use extensively to prove existence and regularity of solutions to nonlinear problems.

Energy estimates. The basic estimate for solutions of the linear wave equation

$$
u_{t t}-\Delta u=0, \quad u(0, x)=u_{0}(x), \quad u_{t}(0, x)=u_{1}(x), \quad x \in \mathbb{R}^{n}
$$


is conservation of energy. To obtain local conservation of energy multiply (2.1) by $u_{t}$,

$$
\partial_{t}\left(\frac{1}{2} u_{t}^{2}+\frac{1}{2}|\nabla u|^{2}\right)-\partial_{i}\left(u_{t} u_{i}\right)=0,
$$

and integrate on a truncated backward light cone ${ }^{1}$,

$$
K_{a}^{b} \stackrel{\text { def }}{=}\left\{(x, t) \in \mathbb{R}^{3} \times[a, b] ; a \leq t \leq b \leq 0,|x| \leq-t\right\},
$$

to obtain

$$
E(u, b)+\frac{1}{\sqrt{2}} \text { flux }(u, a, b)=E(u, a),
$$

where

$$
\begin{aligned}
& E(u, b) \stackrel{\text { def }}{=} \int_{|x| \leq-b} e(u)(x, b) d x, \quad e(u) \stackrel{\text { def }}{=} \frac{1}{2}\left(\left|u_{t}\right|^{2}+\left|\nabla_{x} u\right|^{2}\right), \\
& \operatorname{flux}(u, a, b) \stackrel{\text { def }}{=} \int_{M_{b}^{a}}\left\{\frac{1}{2}\left|\frac{x}{|x|} u_{t}+\nabla u\right|^{2}\right\} d \sigma, \quad M_{a}^{b} \stackrel{\text { def }}{=}\left\{(x, t) \in \mathbb{R}^{3} \times[a, b] ; \quad|x|=-t\right\} .
\end{aligned}
$$

Integrating equation $(2.2)$ on $\mathbb{R}^{n} \times[0, t]$ we obtain global energy conservation

$$
\|u\|_{e}^{2} \stackrel{\text { def }}{=} \int \frac{1}{2} u_{t}^{2}(t)+\frac{1}{2}|\nabla u(t)|^{2}=\int \frac{1}{2} u_{1}^{2}+\frac{1}{2}\left|\nabla u_{0}\right|^{2} .
$$

Higher energy identities can be derived in the same manner:

$$
E\left(\partial^{k} u, b\right)+\frac{1}{\sqrt{2}} f \operatorname{lux}\left(\partial^{k} u, a, b\right)=E\left(\partial^{k} u, a\right), \quad\|u(t)\|_{k, e}^{2} \stackrel{\text { def }}{=} \sum_{0 \leq i \leq k}\left\|\partial^{i} u(t)\right\|_{e}^{2}=\text { constant }
$$

Another basic estimate is the $L^{\infty}$ decay estimate,

$$
|u(x, t)| \leq \frac{c}{t^{\frac{n-1}{2}}}\left(\left|u_{0}\right|_{W^{k+1,1}}+\left|u_{1}\right|_{W^{k, 1}}\right), \quad k=\left[\frac{n-1}{2}\right] .
$$

In three space dimensions it is easy to derive (2.5) using the explicit form of the solution:

$$
u(x, t)=\partial_{t} \frac{1}{4 \pi t} \int_{|x-y|=t} u_{0}(y) d S y+\frac{1}{4 \pi t} \int_{|x-y|=t} u_{1}(y) d S y .
$$

By Gauss's theorem:

$$
\begin{aligned}
\frac{1}{4 \pi t} \int_{|x-y|=t} u_{1}(y) d S y & =\frac{1}{4 \pi t} \int_{|x-y|=t}\left[\bar{n}(y) u_{1}(y)\right] \cdot \bar{n}(y) d S y \\
& =\frac{1}{4 \pi t} \int_{|x-y| \leq t} \operatorname{div}\left(\bar{n}(y) u_{1}(y)\right) d S y
\end{aligned}
$$

where $\bar{n}(y)$ is the unit outward normal. The divergence term can be estimated by

and integration by parts gives

$$
\mid \operatorname{div}\left(\bar{n}(y) u_{1}(y)|\leq| \nabla u_{1}\left|+\frac{2}{|x-y|}\right| u_{1}(y) \mid,\right.
$$

$$
\int_{\mathbb{R}^{n}} \frac{1}{|x-y|}\left|u_{1}(y)\right| d y \leq(n-1) \int_{\mathbb{R}^{n}}\left|\nabla u_{1}(y)\right| d y .
$$

\footnotetext{
${ }^{1}$ We always write local estimates on cones or truncated cones with vertex at the origin. By space time translation these estimates hold on arbitrary cones or truncated cones.
} 
This implies

$$
\left|\frac{1}{4 \pi t} \int_{|x-y|=t} u_{1}(y) d S y\right| \leq \frac{c}{t} \int_{\mathbb{R}^{n}}\left|\nabla u_{1}(y)\right| d y .
$$

Similar estimates for the term involving $u_{0}$ yield equation $(2.5)$ with $n=3$. For general $n$ the reader is referred to [15].

$L^{p}$ estimates. Heuristically, if we interpolate between the $L^{\infty}$ estimate and the energy estimate for the problem,

$$
\begin{aligned}
& u_{t t}-\Delta u=0, \quad x \in \mathbb{R}^{n}, \\
& u(0)=0, \quad \partial_{t} u(0)=u_{1},
\end{aligned}
$$

we obtain that the map $R(t): u_{1} \rightarrow u$ maps $L^{p}$ into $L^{q}$ provided $p=2(n+1) /(n+3)$, and $q=2(n+1) /(n-1)$. The norm of the map $R(t)$ is given by $c t^{-(n-1) /(n+1)}[15]$.

Strichartz estimates. These are estimates involving space-time norms of the solution [26]. They can be derived from the $L^{p}$ bounds given above and a duality argument [5]. Specifically, for the Cauchy problem (2.1) we have

$$
|u|_{L^{q}\left(\mathbb{R}^{n+1}\right)} \leq C\left(\left|u_{0}\right|_{H^{\frac{1}{2}\left(\mathbb{R}^{n}\right)}}+\left|u_{1}\right|_{H^{-\frac{1}{2}}\left(\mathbb{R}^{n}\right)}\right)
$$

for $q=2(n+1) /(n-1)$. The above estimate also holds in the interior of a light cone $K_{a}^{b}$. This follows from finite speed of propagation and extension theorems.

A variant of the above inequality involves homogeneous Besov spaces and is obtained by applying fractional derivatives to the above [5],

$$
|u|_{\dot{B}_{q}^{\frac{1}{2}}\left(\mathbb{R}^{n+1}\right)} \leq C|| u \|_{e} .
$$

Nonhomogeneous equations. For the nonhomogeneous equation,

$$
u_{t t}-\Delta u=h,
$$

solutions can be expressed by the variation of parameters formula

$$
u=u_{\text {lin }}+\int_{0}^{t} R(t-z) h(z) d z .
$$

Estimates can be derived from the previous bounds and Duhamel's principle:

$$
\begin{aligned}
& \sqrt{E(u, b)} \leq C\left(\sqrt{E(u, a)}+\int_{a}^{b}|h(t)|_{L^{2}(D(t))} d t\right), \\
& |u|_{\dot{B}_{q}^{1 / 2}\left(K_{a}^{b}\right)} \leq C\left(\sqrt{E(u, a)}+|h|_{\dot{B}_{p}^{1 / 2}\left(K_{a}^{b}\right)}\right),
\end{aligned}
$$

where $D(s)=\left\{x \in \mathbb{R}^{n} ;|x| \leq|s|\right\}$.

We can combine the energy estimate and the Strichartz estimate by observing that in deriving the energy estimate the space time integral of $h \partial_{t} u$ can be estimated by duality

to obtain

$$
\left.\left|\left\langle h, \partial_{t} u\right\rangle \leq C\right| u\right|_{\dot{B}_{q}^{1 / 2}}|h|_{\dot{B}_{p}^{1 / 2}},
$$

$$
\sqrt{E(u, b)} \leq C\left(\sqrt{E(u, a)}+|h|_{\dot{B}_{p}^{1 / 2}\left(K_{a}^{b}\right)}\right)
$$


3. Semilinear problems. In this section we will establish the existence and uniqueness of smooth solutions and finite energy solutions to the Cauchy problem

$$
\begin{aligned}
& u_{t t}-\Delta u+g(u)=0, \quad x \in \mathbb{R}^{n}, \\
& u(x, 0)=u_{0}(x), \quad u_{t}(x, 0)=u_{1}(x) .
\end{aligned}
$$

3.1. Subcritical case. To prove existence and uniqueness of solutions to nonlinear problems we need to obtain a priori estimates. We'll start by considering a pure power nonlinearity in three space dimensions:

$$
\begin{aligned}
& u_{t t}-\Delta u+|u|^{p-1} u=0, \quad x \in \mathbb{R}^{3}, \\
& u(x, 0)=u_{0}(x), \quad u_{t}(x, 0)=u_{1}(x),
\end{aligned}
$$

and first consider the case $1 \leq p \leq 3$. The simplest way to obtain a priori estimates for (3.1) is to rewrite it using the variation of parameters formula and then apply the standard energy estimate

$$
\|u(t)\|_{e} \leq\left\|u_{\text {lin }}\right\|_{e}+\int_{0}^{t}\left|u^{p}(z)\right|_{2} d z=\left\|u_{\text {lin }}\right\|_{e}+\int_{0}^{t}|u(z)|_{2 p}^{p} d z .
$$

For the case $p=3$ we can use Sobolev embedding,

$$
\left(\int|u|^{6}\right)^{\frac{1}{6}} \leq C\left(\int|\nabla u|^{2}\right)^{\frac{1}{2}},
$$

to rewrite the energy estimate,

$$
\|u(t)\|_{e} \leq\left\|u_{l i n}\right\|_{e}+\int_{0}^{t} C\|u(z)\|_{e}^{3} d z .
$$

If we denote $y(t)=\sup _{0 \leq z \leq t}\|u(z)\|_{e}$, the above equation implies

$$
y(t) \leq\left\|u_{l i n}\right\|_{e}+\operatorname{Cty}(t)^{3} .
$$

Define $T_{*}=\frac{1}{8 C^{3}\left\|u_{l i n}^{2}\right\|_{e}^{2}}$, we obtain

$$
y(t) \leq 2\left\|u_{\text {lin }}\right\|_{e} \quad 0 \leq t \leq T_{*} .
$$

The above estimate is sufficient to prove local existence of solutions with finite energy initial data using a standard contraction mapping argument. The same is true for $1 \leq$ $p \leq 3$ since we can bound $|u|_{L^{2 p}}$ by the energy norm.

To obtain an a priori bound on solution to equation (3.1) that hold for all time multiply the equation by $u_{t}$,

$$
\partial_{t}\left(\frac{1}{2}\left|u_{t}\right|^{2}+\left.\frac{1}{2} \nabla u\right|^{2}+\frac{1}{p+1}|u|^{p+1}\right)-\partial_{i}\left(u_{i} u_{t}\right)=0,
$$

and integrate on $\mathbb{R}^{3} \times[0, t]$ to obtain

$$
\int \frac{1}{2}\left|u_{t}\right|^{2}+\frac{1}{2}|\nabla u|^{2}+\frac{1}{p+1}|u|^{p+1} d=E_{0}
$$

This bound on $\|u(t)\|_{e}$, for all $t$, coupled with a continuation argument proves global existence [24].

THEOREM $3.1(1 \leq p \leq 3)$. Given initial data in $\dot{H}^{1} \times L^{2}$, equation (3.1) has a unique global solution $u \in C\left(\mathbb{R}, \dot{H}^{1}\right)$ and $\partial_{t} u \in C\left(\mathbb{R}, L^{2}\right)$. 
If the initial data is smooth, then so is the solution. The proof of this fact is Gronwall's inequality applied to higher energy estimates.

TheOREm $3.2(1 \leq p \leq 3)$. Given smooth initial data, equation (3.1) has a unique global smooth solution $u$.

For $p>3$ we cannot prove existence and uniqueness using energy estimates alone. However we can prove existence of smooth solutions using the pointwise estimate (2.5). We'll present here an argument that is due to Jörgens [11]. The variation of parameter formula (2.8) in three space dimensions is given by

$$
u(x, t)=u_{l i n}(x, t)+\frac{1}{4 \pi} \int_{0}^{t} \int_{|x-y|=t-s}-\frac{\left|u(y, s)^{p-1}\right|}{t-s} u(y, s) d y d s,
$$

where $u_{\text {lin }}(x, t)$ is the solution of the linear equation with the given initial data. The nonlinear term in (3.7) can be estimated by

$$
\begin{aligned}
\int_{M} \frac{|u|^{p}}{\zeta} d \sigma & \leq\left(\int_{M}|u|^{2(p-1)} d \sigma\right)^{\frac{1}{2}}\left(\int_{M} \frac{u^{2}}{\zeta^{2}} d \sigma\right)^{\frac{1}{2}} \\
& \leq C\left(\int_{M}|u|^{2(p-1)} d \sigma\right)^{\frac{1}{2}}\left[\left(\int_{M}\left|\nabla_{\tan } u\right|^{2}\right)^{\frac{1}{2}}+\text { boundary term }\right]
\end{aligned}
$$

where $M$ is the mantel of the cone with vertex $(x, t)$ and base $t=0, \zeta$ is the distance on the surface of the cone measured from the vertex, and where $\nabla_{t a n}$ are the tangential derivatives to the mantel $M$. The boundary term depends on the initial data only. Integrating the energy identity (3.5) on the solid cone $K$ we obtain

$$
\int_{M}\left|\nabla_{t a n} u\right|^{2} d \sigma \leq C E_{0}
$$

Thus from equations (3.7) and (3.8), the solution $u$ satisfies

$$
|u(x, t)| \leq\left|u_{\text {lin }}(x, t)\right|+C\left(E_{0}\right)\left|u^{p-1}\right|_{L^{2}(M)} .
$$

Since the initial data are smooth we have

$$
\left|u_{\text {lin }}(x, t)\right| \leq C|| u_{l i n} \|_{1, e},
$$

and this implies

$$
|u(x, t)| \leq C|| u_{l i n} \|_{1, e}+C\left(E_{0}\right)\left|u^{p-1}\right|_{L^{2}(M)} .
$$

For $p<4$ the right hand side of the above inequality is bounded by the energy of the initial data, while for $4 \leq p<5$ we can bound the right hand side by

$$
|u(x, t)| \leq C|| u_{\text {lin }} \|_{1, e}+C\left(E_{0}\right)|u|_{L^{\infty}}^{p-4} .
$$

Thus for $1 \leq p<5$ inequality implies a pointwise bound on solutions

$$
|u|_{\infty} \leq C\left(\left\|u_{l i n}\right\|_{1, e}\right) .
$$

Once we have a pointwise bound on the solution we can estimate the solution in any $H^{k}$ space by using higher energy estimates.

THEOREM $3.3(1 \leq p<5)$. Given smooth initial data, equation (3.1) has a unique global smooth solution $u$. 
For finite energy initial data, or for smooth initial data in dimension $n>3$, we can not obtain pointwise bounds directly for the energy bound because of the large number of derivatives needed for such an estimate, i.e. [ $(n-1) / 2]$. An alternative approach to the $L^{\infty}$ estimate is to control Besov norms [5]. We will consider a pure power nonlinearity

$$
\begin{aligned}
& u_{t t}-\Delta u+|u|^{p-1} u=0, \quad x \in \mathbb{R}^{n}, \\
& u(0)=u_{0}, \quad \partial_{t} u(0)=u, \quad p<2^{*}-1=(n+2) /(n-2) .
\end{aligned}
$$

By Duhamel's principle

$$
u(t)=u_{l i n}(t)+\int_{0}^{t} R(t-s)|u(s)|^{p-1} u(s) d s,
$$

where $R$ is the fundamental solution in $n$ space dimensions. Applying Strichartz estimates to the above equation we obtain

$$
|u|_{\dot{B}_{q}^{\frac{1}{2}}\left(\mathbb{R}^{n+1}\right)} \leq|| u_{l i n} \|_{e}+\left.\left.|| u\right|^{p-1} u\right|_{\dot{B}_{q^{\prime}}^{\frac{1}{2}}\left(\mathbb{R}^{n+1}\right)} \leq E_{o}+|u|_{L^{r}\left(\mathbb{R}^{n+1}\right)}^{p-1}|u|_{\dot{B}_{q}^{\frac{1}{2}}\left(\mathbb{R}^{n+1}\right)},
$$

where $q=2(n+1) /(n-1)$ and $r=(n+1)(p-1) / 2$. We can estimate $|u|_{L^{r}\left(\mathbb{R}^{n+1}\right)}$ by the embedding $\dot{B}_{q}^{\frac{1}{2}}\left(\mathbb{R}^{n+1}\right) \subset L^{r}\left(\mathbb{R}^{n+1}\right)$ provided $r=2(n+1) /(n-2)$, i.e. $p=2^{*}-1$. In this case we have

$$
|u|_{\dot{B}_{q}^{\frac{1}{2}}} \leq E_{o}+c|u|_{\dot{B}_{q}^{\frac{1}{2}}}^{p} .
$$

Clearly the above inequality doesn't give an a priori bound on $u$. However if we consider the case $p<2^{*}-1$ and use the local version of Strichartz's estimate we'll obtain

$$
|u|_{\dot{B}_{q}^{\frac{1}{2}}\left(K_{a}^{b}\right)} \leq C\left(E(u, a)+|u|_{L^{r}\left(K_{a}^{b}\right)}^{p-1}|u|_{\dot{B}_{q}^{\frac{1}{2}}\left(K_{a}^{b}\right)}\right) \leq C\left(E(u, a)+|m|^{\alpha}|u|_{\dot{B}_{q}^{\frac{1}{2}}\left(K_{a}^{b}\right)}^{p}\right),
$$

where $m=$ volume of $K_{a}^{b}$ and $\alpha=(n-2) / 2(n+1)-2 /(p-1)(n+1)$. This inequality leads to an estimate on the local $\dot{B}_{q}^{\frac{1}{2}}$ norm of the solution provided we take the cone to have sufficiently small volume.

Therefore to prove existence of solutions to subcritical problems in $\mathbb{R}^{n}$, we set up a contraction mapping argument in the space $\dot{B}_{q}^{\frac{1}{2}}$ on small cones and prove local existence of solutions with finite energy initial data. These solutions satisfy the energy identity which can be derived by multiplying the equation by $\partial_{t} u$ and bounding the nonlinear term by

$$
\left|\left\langle|u|^{p-1} u, u_{t}\right\rangle\right| \leq\left.\left.\left|\partial_{t} u\right|_{\dot{B}_{q}^{-\frac{1}{2}}}|| u\right|^{p-1} u\right|_{\dot{B}_{q^{\prime}}^{\frac{1}{2}}} \leq c\left|\partial_{t} u\right|_{\dot{B}_{q}^{\frac{1}{2}}}|u|_{\dot{B}_{q}^{\frac{1}{2}}}^{p}
$$

where $\langle$,$\rangle denotes space time duality. The energy identity implies a global bound on$ $E(u, a)$ which allows us to use a continuation argument to obtain global existence.

THEOREM $3.4\left(1 \leq p<2^{*}-1\right)$. Given finite energy initial data there exists a unique finite energy solution with the added regularity $u \in \dot{B}_{q}^{\frac{1}{2}}$ and $\partial_{t} u \in \dot{B}_{q}^{\frac{1}{2}}$.

3.2. Critical problems. To solve the wave equation with critical exponent nonlinearity $p=2^{*}-1$,

$$
u_{t t}-\Delta u+|u|^{p-1} u=0, \quad x \in \mathbb{R}^{n},
$$


the Strichartz and the energy estimates (2.9) (2.10) applied to the above equation,

$$
\begin{aligned}
|u|_{\dot{B}_{q}^{\frac{1}{2}}\left(K_{a}^{b}\right)} & \leq C\left(\sqrt{E(u, a)}+|u|_{\dot{B}_{q}^{\frac{1}{2}}\left(K_{a}^{b}\right)}^{p}\right), \\
\sqrt{E(u, b)} & \leq C\left(\sqrt{E(u, a)}+|u|_{\dot{B}_{q}^{\frac{1}{2}}\left(K_{a}^{b}\right)}^{p}\right),
\end{aligned}
$$

fail to give a bound on the Besov space norm unless $E(u, a)$ is sufficiently small. The best bound we have is the total energy bound $E(u, a) \leq E_{0}$. Therefore if the total energy is small the above inequality implies a bound on the Besov norm of $u$.

R e mark 3.1. For smooth initial data in three space dimensions equation (3.11) implies a pointwise bound on solutions provided the initial energy is small. This is the basis of the proof that small energy implies regularity [18]. Moreover on a closer examination of Jörgens proof for the subcritical problem (equations (3.10) and (3.11)) we note that it is sufficient for the nonlinear part of the energy, i.e. $|u(t)|_{L^{6}}$, to be small to obtain a pointwise bound on $u$.

Local existence. Inequalities (3.14) and (3.15) are sufficient to prove local existence and uniqueness of solutions with finite energy initial data. To accomplish this we first start by partitioning $\mathbb{R}^{3}$ into small balls so that on each ball the initial data has small energy. Next we set up a contraction argument on cones $K$ (whose base is one of these balls) in the space

$$
u \in \dot{B}_{q}^{\frac{1}{2}}(K) \cap L^{\infty}\left(\dot{H}^{1}\right), \quad \partial_{t} u \in \dot{B}_{q}^{-\frac{1}{2}}(K) \cap L^{\infty}\left(L^{2}\right) .
$$

By finite propagation speed we can put these solutions together to generate a local solution for the given initial data $[12,21]$.

THEOREM 3.5. Given finite energy initial data equation (3.13) has a unique local solution in the space given by (3.16).

Global existence. In order to prove that the local solutions can be extended globally we have to show that the nonlinear part of the energy can't concentrate at a point. Without loss of generality we consider the point $(0,0)$ and use the local conservation of energy and the Morawetz identity.

Lemma 3.1. Given a finite energy solution $u \in \dot{B}_{q}^{\frac{1}{2}}\left(K_{a}^{b}\right) \forall b>a$, then

(a) $\operatorname{flux}(u, 0, a) \rightarrow 0$ as $a \rightarrow 0$,

(b) $\int_{D(a)}|u(a)|^{2^{*}} \rightarrow 0$ as $a \rightarrow 0$.

One of the difficulties in proving this lemma is the derivation of the energy and the Morawetz identities. Since the solution is assumed to be of finite energy only, some care has to be taken in multiplying equation (3.13) by derivatives of $u$, as required in deriving these identities. The linear part of the equation can be dealt with in a standard manner such as smoothing the solution by an approximate identity $u_{j}=\varphi_{j} * u$. Moreover since $u$ is assumed to be in $\dot{B}_{q}^{\frac{1}{2}}\left(K_{a}^{b}\right)$ we have the nonlinear term $|u|^{p-1} u \in \dot{B}_{q^{\prime}}^{\frac{1}{2}}\left(K_{a}^{b}\right)$. Therefore multiplying the nonlinear term by derivatives of $u$ and integrating over space time regions is admissible by duality. To localize the identities we multiply by the cutoff function $\eta$ on 
the cone $K$

$$
\eta= \begin{cases}1, & t+x \leq-\varepsilon, t_{o}+\varepsilon<t<0, \\ 1-\frac{t+|x|+\varepsilon}{\varepsilon}, & -\varepsilon \leq t+|x| \leq 0, \frac{1}{2} t_{o} \leq t+\frac{1}{2}|x|, \\ 1+\frac{t-t_{o}-\varepsilon}{\varepsilon}, & t_{o} \leq t \leq t_{o}+\varepsilon, t+\frac{1}{2}|x| \leq \frac{1}{2} t_{o} .\end{cases}
$$

For example to derive the energy identity we multiply equation (3.13) by $\eta \partial_{t} u_{j}$. Integrating by parts and letting $j \rightarrow \infty$ we obtain

$$
g(\varepsilon)=\iint\left\{\eta_{t}\left(\frac{1}{2}\left|u_{t}\right|^{2}+\frac{1}{2}|\nabla u|^{2}+\frac{1}{2^{*}}|u|^{2^{*}}\right)-\nabla \eta \cdot\left(\nabla u u_{t}\right)\right\} d x d t=0,
$$

Computing $d(\varepsilon g) / d \varepsilon$ we obtain local energy conservation

$$
E(u, b)+\frac{1}{\sqrt{2}} \operatorname{flux}(u, a, b)=E(u, a),
$$

where

$$
\begin{aligned}
& e(u) \stackrel{\text { def }}{=} \frac{1}{2}\left(\left|u_{t}\right|^{2}+\left|\nabla_{x} u\right|^{2}\right)+\frac{1}{2^{*}}|u|^{2^{*}} \\
& \operatorname{flux}(u, a, b) \stackrel{\text { def }}{=} \int_{M_{b}^{a}}\left\{\frac{1}{2}\left|\frac{x}{|x|} u_{t}+\nabla u\right|^{2}+\frac{1}{2^{*}}|u|^{2^{*}}\right\} d \sigma .
\end{aligned}
$$

The boundedness of the flux in equation (3.17) implies part a) of the lemma.

The Morawetz identity can be derived in a similar fashion using the multiplier

$$
\eta\left(t \partial_{t} u_{j}+x \cdot \nabla u_{j}+(n-2) u_{j} / 2\right)
$$

and integrating over $K_{a}^{b}$, letting $b \rightarrow 0$ we obtain

$$
-\int_{D(a)} a Q_{0}+(n-1) u u_{t} / 2+\int_{K} R_{0}+\frac{1}{\sqrt{2}} \int_{C}\left\{t Q_{0}+\frac{n-1}{2} u_{t} u+x \cdot P_{0}\right\}=0 .
$$

After some algebraic manipulation and integration by parts the above equation yields $[20]$

$$
-a \int_{D(a)}|u|^{2^{*}} \leq c|a| \operatorname{flux}(u, 0, a) .
$$

Dividing by $-a$ and letting $a \rightarrow 0$ proves part b) of the lemma.

To obtain bounds on the $\dot{B}^{\frac{1}{2}}\left(K_{a}^{b}\right)$ norm of the solution $u$ in inequality (3.12)

$|u|_{\dot{B}_{q}^{\frac{1}{2}}\left(K_{a}^{b}\right)} \leq E(u, a)+|u|_{L^{r}\left(K_{a}^{b}\right)}^{p-1}|u|_{\dot{B}^{\frac{1}{2}}\left(K_{a}^{b}\right)}, \quad q=2(n+1) /(n-1), r=2(n+1) /(n-2)$.

we estimate the $L^{r}\left(K_{a}^{b}\right)$ norm of $u$ as follows: By Hölder's inequality and the embedding $\dot{B}_{q}^{\frac{1}{2}}\left(\mathbb{R}^{n}\right) \subset L^{s}\left(\mathbb{R}^{n}\right)$ we can estimate the spatial norm of $u$,

$$
\begin{gathered}
|u(t)|_{r} \leq|u(t)|_{L^{2^{*}(D(t))}}^{\alpha}|u(t)|_{L^{s}(D(t))}^{1-\alpha} \leq c|u(t)|_{L^{2^{*}(D(t))}}^{\alpha}|u(t)|_{\dot{B}_{q}^{\frac{1}{2}}}^{1-\alpha}, \\
\alpha=1 /(n-1), 1 / s=(n-1) / 2(n+1)-1 / 2 n .
\end{gathered}
$$


The space time norm of $u$ can be now estimated by

$$
|u|_{L^{r}\left(K_{a}^{b}\right)}^{r} \leq C \int_{a}^{b}|u(t)|_{L^{2^{*}(D(t))}}^{\alpha r}|u(t)|_{\dot{B}_{q}^{\frac{1}{2}}(D(t))}^{(1-\alpha) r} d t \leq c|u|_{L^{\infty}\left(L^{\left.2^{*}\right)}\right)}^{\alpha r}|u|_{\dot{B}_{q}^{\frac{1}{2}}\left(K_{a}^{b}\right)}^{q},
$$

since $(1-\alpha) r=q$. This leads to the inequality

$$
|u|_{\dot{B}_{q}^{\frac{1}{2}}\left(K_{a}^{b}\right)} \leq E_{0}+c|u|_{L^{\infty}\left(L^{2^{*}}\right)}^{p-\gamma}|u|_{\dot{B}^{\frac{1}{2}}\left(K_{a}^{b}\right)}^{\gamma}, \quad \gamma=(n+3) /(n-1),
$$

which implies a bound on the Besov norm of $u$ provided the $L^{\infty}\left(L^{2^{*}}(D(t))\right)$ norm of $u$ is small on $K_{a}^{b}$. The decay of the $L^{2^{*}}$ of $u$ and equation (3.19) implies that $u \in \dot{B}_{q}^{\frac{1}{2}}(K)$. To show that the whole energy decays apply estimate $(2.10)$ to $u-u_{l i n}$,

$$
\sqrt{E\left(u-u_{\text {lin }}\right)} \leq c|u|_{\dot{B}_{q}^{\frac{1}{2}}\left(K_{a}^{b}\right)}^{p}, \quad a \leq b \leq 0 .
$$

Since $u_{l i n}$ is a solution of the linear equation and $|u|_{\dot{B}_{q}^{\frac{1}{2}}(K)} \leq C$, then $E(u, b)$ can be made arbitrarily small for $a \leq b \leq 0$ by taking the cone $K$, i.e. $a$, to be small.

The decay of $E(u, b)$ at the tip of the cone allows us to extend the local solution to a global one. For let $u$ be a maximal solution defined on $\mathbb{R}^{n} \times[T, 0)$ and $K$ a cone with vertex at $t=0$. Since the energy decays on the cone $K$ we have $E(u, b) \rightarrow 0$ as $b \rightarrow 0$. Take $b$ small enough so that the energy in a ball with center at $(0, b)$, and radius slightly larger then $|b|$ is smaller then what is required by the local existence theorem. Then the solution can be extended beyond the tip of $K[12,21]$.

THEOREM $3.6\left(p \leq 2^{*}-1\right)$. Given finite energy initial data equation (3.13) has a unique global solution.

4. Wave maps. An instructive way to describe the problem is when the target manifold $N$ is a hypersurface in $\mathbb{R}^{k+1}$. In this case if $u(s) \in N \subset \mathbb{R}^{k+1}$ is a curve on $N$ and $V(s)$ is a tangent vector to $N$ at $u(s)$ then the covariant derivative $D_{s} V(s)$ is given by

$$
D_{s} V(s)=\frac{d}{d s} V(s)+\left(V(s) \cdot \frac{d}{d s} n(s)\right) n(s),
$$

where $n(s)$ is the unit normal vector to $N$ at $u(s)$. Thus if we use $\left(x^{0}, x^{1}, \ldots, x^{n}\right)=(t, x)$ as a coordinate system on $\mathbb{R}^{n+1}$ with the metric $\left(\eta_{\alpha \beta}\right)=(-1,1, \ldots, 1)$ and consider a map $u: \mathbb{R}^{n+1} \rightarrow N \subset \mathbb{R}^{k+1}$, then $\partial_{\alpha} u$ is a tangent vector to $N$ at $u$ and

$$
D_{\beta} \partial_{\alpha} u=\partial_{\beta} \partial_{\alpha} u+\left(\partial_{\alpha} u \cdot \partial_{\beta} n(u)\right) n(u) \text {. }
$$

Consequently the wave equation of the map $u$ is given by $\eta^{\alpha \beta} D_{\beta} \partial_{\alpha} u=0$ or

$$
\partial_{0}^{2} u-\Delta u+\left(\partial_{0} u \cdot \partial_{0} n(u)-\partial_{i} u \cdot \partial_{i} n(u)\right) n(u)=0,
$$

where we use the summation convention on repeated indices.

In general for a complete Riemannian manifold $(N, g)$ and a map $u$ : $\mathbb{R}^{n+1} \rightarrow N$ we consider the Cauchy problem

$$
\begin{aligned}
& D_{\alpha} \partial \alpha u^{a}=\partial^{\alpha} \partial_{\alpha} u^{a}+\Gamma_{b c}^{a}(u) \partial_{\alpha} u^{b} \partial^{\alpha} u^{c}=0, \\
& u(0, x)=U_{0}(x), \quad \partial_{t} u(0, x)=U_{1}(x),
\end{aligned}
$$


where $\Gamma$ are the Christoffel symbols of the metric $g$. We will obtain sharp results on existence and uniqueness for equivariant maps for space dimensions greater than two.

4.1. Conserved quantities. Equation 4.1 has a variational formulation

$$
\mathcal{A}(u)=\int L(u) d v=\int \frac{1}{2} g_{a b}(u) \partial_{\alpha} u^{a} \partial_{\beta} u^{b} \eta^{\alpha \beta} d v .
$$

By Noether's Theorem any one parameter group that leaves the action invariant generates a conserved current. Thus we have the usual energy-momentum conservation from space time translation and we have conserved currents generated by the isometry of $N$. We'll illustrate this by considering the case $N=\mathbb{S}^{2} \subset \mathbb{R}^{3}$. In this case the unit normal to $\mathbb{S}^{2}$ at a point $u \in \mathbb{R}^{3}$ is $u$, and the wave equation for $u \in \mathbb{S}^{2} \subset \mathbb{R}^{3}$ is

$$
\begin{aligned}
& u_{t t}-\Delta u+\left(\left|u_{t}\right|^{2}-|\nabla u|^{2}\right) u=0, \\
& u(0, x)=u_{0}(x), \quad \partial_{t} u(0, x)=u_{1}(x) .
\end{aligned}
$$

Invariance under time translation generates conservation of energy

$$
\partial_{0}\left(\frac{1}{2}\left|u_{t}\right|^{2}+\frac{1}{2}|\nabla u|^{2}\right)-\partial_{i}\left(u_{i} \cdot u_{t}\right)=0,
$$

while invariance under space translation generates conservation of momentum

$$
\partial_{0}\left(u_{k} \cdot u_{t}\right)-\partial_{i}\left(\frac{1}{2}\left|u_{t}\right|^{2}+u_{k} \cdot u_{i}-\frac{1}{2} \delta_{i k}|\nabla u|^{2}\right)=0 .
$$

Now if we consider the invariance of the action under rotation of $u$ on in $S^{2}$ we have the conserved current

$$
\partial_{0}\left(u_{t} \wedge u\right)-\partial_{i}\left(u_{i} \wedge u\right)=0,
$$

where $\wedge$ is the wedge product.

For the general case, energy-momentum conservation can be computed using the energy-momentum tensor which is the variation of the action with respect to the metric on $\mathbb{R}^{n+1}$

$$
T_{\alpha \beta}=\frac{\delta \mathcal{A}}{\delta \eta^{\alpha \beta}}=g_{a b} \partial_{\alpha} u^{a} \partial_{\beta} u^{b}+\frac{1}{2} \eta_{\alpha \beta} L(u),
$$

and energy momentum conservation can be expressed as

$$
\partial_{\alpha}\left(T^{\alpha \beta}\right)=0
$$

Conserved current generated by isometries of $N$ can be obtained by computing the variation of the action with respect to the metric on the target. Let $\phi_{\epsilon}$ denote a one parameter family of diffeomorphisms generated by a vector field $X$, and $g^{\epsilon}$ the pullback of the metric $g$ by $\phi$, i.e.

Then

$$
g_{a b}^{\epsilon}(u)=g_{c d}\left(\phi_{\epsilon}(u)\right) \frac{\partial \phi_{\epsilon}^{c}}{\partial u^{a}} \frac{\partial \phi_{\epsilon}^{d}}{\partial u^{b}} .
$$

$$
\left.\frac{d \mathcal{A}}{d \epsilon}\right|_{\epsilon=0}=\frac{1}{2} \int\left(\mathcal{L}_{x} g\right)_{a b} \partial_{\alpha} u^{a} \partial_{\beta} u^{b} \eta^{\alpha \beta} d v,
$$

where $\left(\mathcal{L}_{x} g\right)_{a b}=D_{a} X_{b}+D_{b} X_{a}$ is the Lie derivative. Moreover since $D_{a} X_{b} \partial_{\alpha} u^{a}=$ $D_{\alpha} X_{b}(u)$, equation (4.2) can be written as

$$
\left.\frac{d \mathcal{A}}{d \epsilon}\right|_{\epsilon=0}=\int D_{\alpha}\left(\eta^{\alpha \beta} X_{b}(u) \partial_{\beta} u^{b}\right)-\eta^{\alpha \beta}\left(X_{b} D_{\alpha} \partial_{\beta} u^{b}\right) .
$$


If $X$ generates an isometry on $N\left(\frac{d \mathcal{A}}{d \epsilon}=0\right)$, and if $u$ is a solution to 4.1 , we obtain the conserved current

$$
D_{\alpha}\left(\eta^{\alpha \beta} X_{b}(u) \partial_{\beta} u^{b}\right)=0
$$

4.2. Local existence. Equations (4.1) are semilinear wave equations, and therefore there is no problem in proving local existence and uniqueness of solutions for smooth initial data. However if we pose the Cauchy problem with initial data in $H^{s}$ for small $s$ then the problem is much more difficult. Since the equations are invariant under the scaling $u_{\lambda}(t, x)=u(\lambda t, \lambda x)$ it is easy to see that, in general, the problem cannot be well-posed in $H^{s}$ for $s<\frac{n}{2}$. This can be seen by noting that any solution that blows up in finite time, can be scaled so that the $H^{s}$ norm of the Cauchy data approaches zero and the existence time approaches zero as well. This implies that solutions do not depend continuously on the initial data since the zero solution exists for all time. (See [14] for examples where similar scaling argument fails to give the best possible space for local existence.)

As stated in the introduction, there is no proof that $H^{\frac{n}{2}}$ is the optimal space for local existence. Here we will give a simple proof, based on energy estimates only, that the Cauchy problem is well-posed in $H^{\frac{n+1}{2}}$ for $n \geq 3$. For $n=3$ and $N=\mathbb{S}^{k}$ this result was proved by M. Struwe [28]. Moreover, for general quadratic nonlinearities involving derivatives, G. Ponce and T. Sideris [17] proved that the Cauchy problem is well-posed in three space dimensions in the space $H^{2+\varepsilon}$.

THEOREM 4.1. The Cauchy problem (4.1) is well posed in the space $H^{\frac{n+1}{2}}$ for $n \geq 3$.

Sketch of the proof. We start our proof by deriving higher energy estimates for equation (4.1). Let $V_{\alpha}=\partial_{\alpha} u$ then equation (4.1) can be written as $\eta^{\alpha \beta} D_{\alpha} V_{\beta}=0$. Differentiating with respect to $x^{\gamma}$ we obtain

$$
\begin{aligned}
& \eta^{\alpha \beta} D_{\alpha} D_{\beta} V_{\gamma}+\eta^{\alpha \beta} R\left(\partial_{\gamma} u, \partial_{\alpha} u\right) V_{\beta}=0, \\
& V_{\gamma}(0)=V_{\gamma}, \quad D_{0} V_{\gamma}(0)=V_{1} .
\end{aligned}
$$

Multiplying by $D_{0} V_{\gamma}$ and integrating by parts yields

$$
\frac{d}{d t} \sqrt{E(V)} \leq C\left|\eta^{\alpha \beta} R\left(\partial_{\alpha} u, \partial_{\gamma} u\right) V_{\beta}\right|_{L^{2}}
$$

where $V=\left(V_{0}, \ldots, V_{n}\right)$ and $E(V)=\int \frac{1}{2}\left|D_{0} V\right|^{2}+\frac{1}{2} \Sigma\left|D_{i} V\right|^{2}$ is the energy of $V$. By Sobolev embedding the right hand side of the above inequality can be estimated by

$$
\frac{d}{d t} \sqrt{E(V)} \leq C|\partial u|_{L^{2 n}}^{2}|V|_{H^{1}} \leq C|u|_{H^{\frac{n+1}{2}}}^{2} \sqrt{E(V)}
$$

By Gronwall's Lemma we obtain a bound on $E(V)$ in terms of the $H^{\frac{n+1}{2}}$ norm of $u$,

$$
E(V(t)) \leq \exp \left[C \int_{0}^{t}|u|_{H^{\frac{n+1}{2}}}^{2}\right] E(V(0))
$$

Higher energy estimates can be obtained in the same manner by differentiating equation (4.3) $k$ times and multiplying by $D_{0} D^{k} V$. All the commutator terms as well as the curvature terms are lower order and can be bounded by the norm of $u$ in $H^{\frac{n+1}{2}}$ and $E\left(D^{k} V\right)$. Consequently if $n$ is odd we obtain a priori estimates on the $H^{\frac{n+1}{2}}$ norm of 
$u$ in terms of the initial data. If $n$ is even we obtain the same estimate using interpolation. This is carried out in the following manner: For any $u \in H^{\frac{n+1}{2}}$ consider the linear equation

$$
\eta^{\alpha \beta} D_{\alpha} D_{\beta} W=0, \quad W(0)=W_{0}, \quad D_{0} W(0)=W_{1} .
$$

Then from energy estimates for $W$ we have

$$
|W(t)|_{H^{1}} \leq C\left(|u|_{H^{\frac{n+1}{2}}}\right)|W(0)|_{H^{1}}, \quad|W(t)|_{H^{2}} \leq C\left(|u|_{H^{\frac{n+1}{2}}}\right)|W(0)|_{H^{2}} .
$$

By interpolation we obtain

$$
|W(t)|_{H^{\frac{3}{2}}} \leq C\left(|u|_{H^{\frac{n+1}{2}}}\right)|W(0)|_{H^{\frac{3}{2}}} .
$$

Therefore for $n$ even we can differentiate the equation $\frac{n-2}{2}$ times and then apply the above estimate to obtain $H^{\frac{n+1}{2}}$ bound on the solution. These a priori estimates are used to obtain existence of $H^{\frac{n+1}{2}}$ solution as limits of smooth solutions. The local existence time will depend only on the $H^{\frac{n+1}{2}}$ norm of the initial data.

To show uniqueness we need to compare two solutions, say $u_{1}$ and $u_{2}$ with the same initial data. However since solutions are maps into manifolds we cannot simply consider the difference $u_{1}-u_{2}$. We get around this difficulty by considering the tangent vectors $V_{\alpha}=\partial_{\alpha} u_{1}$ and $W_{\alpha}=\partial_{\alpha} u_{2}$. Since $u_{1}$ and $u_{2}$ are Hölder continuous and coincide at $t=0$ we have for $t$ sufficiently small $u_{1}(x)$ and $u_{2}(x)$ in a geodesically convex region where $u_{1}(x)$ and $u_{2}(x)$ can be connected by a unique geodesic $\gamma(s, x)$ of length $\ell(x)$, i.e. $\gamma(0, x)=u_{1}(x), \gamma(\ell(x), x)=u_{2}(x)$ and $D_{s} \partial_{s} \gamma=0$. Let $\tilde{V}(s, x)$ be the parallel translation of $V(x)$ along $\gamma$,

$$
D_{s} \tilde{V}_{\alpha}=0, \quad \tilde{V}_{\alpha}(0, x)=V_{\alpha}(x) .
$$

Then $\tilde{V}_{\alpha}(s, x)=\underline{\bar{X}}(s, 0, x) V_{\alpha}(x)$, where $\underline{\bar{X}}$ is the fundamental matrix solution of the above ODE. Therefore to compare the two solutions $u_{1}$ and $u_{2}$ we compare $\bar{V}_{\alpha}$ and $W_{\alpha}$, where $\bar{V}_{\alpha}(x)=\tilde{V}_{\alpha}(\ell(x), x)$ the parallel translation of $V_{\alpha}(x)$ to the point $u_{2}$ along $\gamma$. Note that the derivative of $\bar{V}_{\alpha}$ can be computed using the equation

$$
\begin{aligned}
& D_{s} D_{\beta} \tilde{V}_{\alpha}+R\left(\partial_{s} \gamma, \partial_{\beta} \gamma\right) \tilde{V}_{\alpha}=0, \\
& D_{\beta} \bar{V}_{\alpha}=D_{\beta} V_{\alpha}-\int_{0}^{\ell} \underline{X}(\ell, s, x) R\left(\partial_{s} \gamma, \partial_{\beta} \gamma\right) \tilde{V}_{\alpha} .
\end{aligned}
$$

Since $D_{\alpha} V_{\alpha}=0$ and $D_{\beta} V_{\alpha}=D_{\alpha} V_{\beta}$ we obtain

$$
\left|D_{\alpha} \bar{V}_{\alpha}\right| \leq C \ell|V|^{2}, \quad\left|D_{\alpha} \bar{V}_{\beta}-D_{\beta} \bar{V}_{\alpha}\right| \leq C \ell|V|^{2},
$$

where the constant $C$ depends only on the local geometry of the target manifold. Using the above inequalities we can derive $L^{2}$ estimates for $\bar{V}-W$ from the equation

$$
\left|D_{\alpha}\left(\bar{V}_{\alpha}-W_{\alpha}\right)\right| \leq C \ell|V|^{2},
$$

which implies the estimate

$$
\frac{d}{d t}|\bar{V}-W|_{L^{2}} \leq\left.\left. C|\ell| V\right|^{2}\right|_{L^{2}} \quad \leq C|\nabla \ell|_{L^{2}}|V|_{L^{2 n}}^{2} .
$$

Using the first variation of arc length we have $|\nabla \ell| \leq C|\bar{V}-W|$ and this implies the 
inequality

$$
\frac{d}{d t}|\bar{V}-W|_{L^{2}} \leq C|\bar{V}-W|_{L^{2}}|u|_{H^{\frac{n+1}{2}}}^{2}
$$

By Gronwall's Lemma we obtain uniqueness of solutions based on $H^{\frac{n+1}{2}}$ norm of the initial data.

THEOREM 4.2. The Cauchy problem (4.1) with initial data

$$
U_{0} \in H^{\frac{n+1}{2}}, \quad U_{1} \in H^{\frac{n-1}{2}},
$$

has a unique local solution on $\left[0, T^{*}\right)$ for some $T^{*}>0$ :

$$
u \in C\left(\left[0, T_{0}\right], H^{\frac{n+1}{2}}\right), \quad \partial_{0} u \in C\left(\left[0, T_{0}\right], H^{\frac{n-1}{2}}\right), \quad \forall T_{0}<T^{*} .
$$

R e mark 4.1. In one space dimension equation (4.5) implies a global bound for the $H^{2}$ norm of the solution. Higher energy estimates imply that the solution is regular for all time [4].

4.3. Equivariant maps. In this section we will restrict the target manifold to be rotationally symmetric, i.e.

$$
N=\left[0, \phi^{*}\right) \times \mathbb{S}^{k-1},
$$

where $\phi^{*} \in \mathbb{R}^{+} \cup\{+\infty\}$. On $N$ we have polar coordinates $(\phi, \chi) \in\left[0, \phi^{+}\right) \times \mathbb{S}^{k-1}$ and the metric in these coordinate systems can be written as

$$
d s^{2}=d \phi^{2}+g^{2}(\phi) d \chi^{n},
$$

where $g(0)=0, g^{\prime}(0)=1$, and $d \chi^{2}$ is the standard metric on $\mathbb{S}^{k-1}$. For such manifolds there exist a special class of maps called equivariant maps. Using polar coordinates $(t, r, \omega)$ on $\mathbb{R}^{n+1}$, equivariant maps are given by

$$
(t, r, \omega) \rightarrow(\phi(t, r), \chi(\omega))
$$

where $\chi(\omega)$ is a map from $\mathbb{S}^{n-1}$ into $\mathbb{S}^{k-1}$. For equivariant maps to be wave maps $\chi(\omega)$ has to be a harmonic polynomial of degree $\ell$, i.e., the restriction of a map from $\mathbb{R}^{n} \rightarrow \mathbb{R}^{k}$ where each component is a homogeneous polynomial of some degree $\ell>0$, and $\phi$ has to satisfy the Cauchy problem

$$
\begin{aligned}
& \phi_{t t}-\phi_{r r}-\frac{n-1}{r} \phi_{r}+\frac{k}{r^{2}} f(\phi)=0, \\
& \phi(0, r)=\phi_{0}(r), \quad \partial_{t} \phi(0, r)=\phi_{1}(r),
\end{aligned}
$$

with $f(\phi)=g(\phi) g^{\prime}(\phi)$ and $k=\ell(\ell+n-2)$.

The above equation is easier to analyze than (4.1) since the nonlinearity has a singularity at $r=0$ instead of having a product of derivatives. The energy identity for the above equations is given by

$$
E(\phi) \stackrel{\text { def }}{=} \int \frac{1}{2}\left(\phi_{t}^{2}+\phi_{r}^{2}+\frac{k}{r^{2}} g^{2}(\phi)\right) r^{n-1} d r=\int \frac{1}{2}\left(\phi_{1}^{2}+\phi_{0 r}^{2}+\frac{k}{r^{2}} g^{2}\left(\phi_{0}\right)\right) r^{n-1} d r .
$$

As in section 4.2 we can use the scaling invariance of the equations to deduce which problem we can study using conservation of energy only. The equation is invariant under scaling $\phi \rightarrow \phi_{\lambda}(t, r)=\phi(t / \lambda, r / \lambda)$ and the energy $E$ scales as $E\left(\phi_{\lambda}\right)=\lambda^{n-2} E(\phi)$. For $n=1 E\left(\phi_{\lambda}\right) \rightarrow \infty$ as $\lambda \rightarrow 0$, and this rules out concentration since the energy is conserved 
(indeed solutions are regular as remarked in section 4.2), and for $n \geq 3 E\left(\phi_{\lambda}\right) \rightarrow 0$ as $\lambda \rightarrow 0$, which doesn't rule out concentration and development of singularities (which we will show in section 4.4). For $n=2$, existence of singularities is still an open problem and there are only partial results on regularity of solutions.

Local existence in $H^{\frac{n}{2}}$. To prove local existence and uniqueness to (4.6) in the space $H^{\frac{n}{2}}\left(\mathbb{R}^{n}\right)$ we use Strichartz estimates coupled to energy estimates. Let $\phi=r v$, then $v$ satisfies

$$
\begin{aligned}
& v_{t t}-v_{r r}-\frac{m-1}{r} v_{r}=v^{3} Z(r v), \\
& v(0, r)=v_{0}=\phi_{0} / r, \quad v_{t}(0, r)=v_{1}=\phi_{1} / r,
\end{aligned}
$$

where $m=n+2$ and $Z$ is a smooth, even function. It is not difficult to prove the equivalence of the Cauchy problem (4.6) for $\phi$ in $H^{\frac{n}{2}}\left(\mathbb{R}^{n}\right)$ and the Cauchy problem for $v$ (4.7) with initial data

$$
v_{0} \in H_{\mathrm{loc}}^{k}\left(\mathbb{R}^{m}\right), \quad v_{1} \in H_{\mathrm{loc}}^{k-1}\left(\mathbb{R}^{m}\right), \quad k=\frac{m-2}{2} .
$$

This can be done by noting that for radial functions Hardy's inequality and Sobolev embedding theorems imply

$$
\left|r^{\frac{m}{2}-\frac{m}{p}-s} v\right|_{L^{p}\left(\mathbb{R}^{m}\right)} \leq c|v|_{H^{s}\left(\mathbb{R}^{m}\right)},
$$

with $1 \leq s \leq \frac{m}{2}$ and $q \leq p$.

Strichartz estimates. The Besov norm estimate (2.9) applied to equation (4.7) yields

$$
|v|_{\dot{B}_{q}^{s}\left(K_{a}^{b}\right)} \leq c E\left(\partial^{s-\frac{1}{2}} v, a\right)+c\left|v^{3} Z(r v)\right|_{\dot{B}_{q^{\prime}}^{s}\left(K_{a}^{b}\right)},
$$

where $q=2(m+1) /(m-1)$ and where we set $s=k-\frac{1}{2}=(m-3) / 2$. The term $Z(r v)$ in the above inequality will cause no difficulty in carrying out our estimates since, by (4.8),

$$
|r v|_{L^{\infty}} \leq c|v|_{H^{k}}, \quad|v|_{L^{m}} \leq c|v|_{H^{k}}, \quad\left|r \partial^{j} v\right|_{L^{\frac{m}{j}}} \leq c|v|_{H^{k}} .
$$

Therefore the term $v^{3} Z(r v)$ behaves effectively as a cubic term and we only need to estimate $v^{3}$ in $\dot{B}_{q^{\prime}}^{s}\left(K_{a}^{b}\right)$. This can be done in a manner similar to (3.2):

$$
\left|v^{3}\right|_{\dot{B}_{q^{\prime}}^{s}\left(K_{a}^{b}\right)} \leq c|v|_{\dot{B}_{q}^{s}\left(K_{a}^{b}\right)}^{\alpha} \llbracket v \rrbracket^{\beta}, \quad \llbracket v \rrbracket \stackrel{\text { def }}{=} \sup _{t \in[a, b]} \sqrt{E\left(\partial^{s-\frac{1}{2}} v, t\right)},
$$

where $\alpha=(m+3) /(m-1)$ and $\beta=(2 m-b) /(m-a)$. Inequality (4.9) can be estimated by

$$
|v|_{\dot{B}_{q}^{s}\left(K_{a}^{b}\right)} \leq c E\left(\partial^{s-\frac{1}{2}} v, a\right)+c\left(\llbracket v \rrbracket+|v|_{\dot{B}_{q}^{s}\left(K_{a}^{b}\right)}\right)^{m+2} .
$$

Energy estimates. Applying estimate (2.10) to equation (4.7) on a truncated cone $K_{a}^{b^{\prime}}$ for $b^{\prime} \leq b$

$$
\sqrt{E\left(\partial^{s-\frac{1}{2}} v, b^{\prime}\right)} \leq c \sqrt{E\left(\partial^{s-\frac{1}{2}} v, a\right)}+c\left|v^{3} Z(r v)\right|_{\dot{B}_{q^{\prime}}^{s}\left(K_{a}^{b}\right)} .
$$

This yields, as in (4.9),

$$
\llbracket v \rrbracket \leq c \sqrt{E\left(\partial^{s-\frac{1}{2}} v, a\right)}+c\left(\llbracket v \rrbracket+|v|_{\dot{B}_{q}^{\frac{1}{2}}\left(K_{a}^{b}\right)}\right)^{m+2} .
$$


By considering small truncated cone $K_{0}^{b}$ we can make the initial data $E\left(\partial^{s-\frac{1}{2}} v, 0\right)$ small. In this cases the Besov norm estimate (4.10) and the energy estimate (4.11) yield an a priori bound

$$
\llbracket v \rrbracket+|v|_{\dot{B}_{q}^{s}\left(K_{0}^{b}\right)} \leq c \sqrt{E\left(\partial^{s-\frac{1}{2}} v, 0\right)} .
$$

This a priori bound and a standard contraction mapping argument proves

THEOREM 4.3. The Cauchy problem (4.6) with initial data

$$
\phi \in H^{n / 2}, \quad \phi_{1} \in H^{(n-2) / 2},
$$

has a unique local solution on $\left[0, T^{*}\right)$ for some $T^{*}>0$ :

$$
\begin{aligned}
& \phi \in L^{\infty}\left(\left[0, T_{0}\right], H^{n / 2}\right) \cap \dot{B}_{q}^{\frac{1}{2}}\left(\left[0, T_{0}\right] \times \mathbb{R}^{n}\right), \\
& \partial_{t} \phi \in L^{\infty}\left(\left[0, T_{0}\right], H^{n / 2-1}\right), \quad q=\frac{2(n+3)}{n+1}, \quad \forall T_{0}<T^{*} .
\end{aligned}
$$

Global regularity in two space dimensions. Since the problem is critical in two space dimensions, the proof of regularity (as in the semilinear problem) proceeds in two steps: 1 ) small energy implies regularity and 2) energy cannot concentrate. Complete details of the proof can be found in $[8,23]$.

THEOREM $4.4(n=2)$. Let the function $g$ in equation (2) satisfy the condition

$$
g(s)+s g^{\prime}(s)>0 \text { for } s>0 .
$$

Then the Cauchy problem with smooth initial data has smooth solution for all time.

Sketch of the proof. Without loss of generality we will assume that the data is given at $t=-1$ and the first possible singularity is at $(0,0)$. To show that the energy cannot concentrate at $(0,0)$ we will use the energy identity

$$
(r e)_{t}-(r m)_{r}=0,
$$

where $e=\frac{1}{2}\left(\phi_{t}^{2}+\phi_{r}^{2}+\frac{\ell^{2}}{r^{2}} g^{2}(\phi)\right)$ and $m=\phi_{t} \phi_{r}$. We also use a variant of the Morawetz identity which can be derived by multiplying (4.6) by $M_{a} \phi \stackrel{\text { def }}{=} r^{a} \phi_{r}+\frac{1-a}{2} r^{a-1} \phi$ :

$$
\begin{aligned}
\partial_{t}\left[r^{a+1}\left(m+\frac{1-a}{2} \phi_{t} \frac{\phi}{r}\right)\right] & -\partial_{r}\left[r^{a+1}\left(e-\frac{2 \ell^{2}}{r^{2}} g^{2}(\phi)+\phi_{r} \frac{\phi}{r}+\frac{(1-a)^{2}}{4} \frac{\phi^{2}}{r^{2}}\right]\right. \\
= & -a \phi_{t}^{2} r^{a}-\frac{1-a}{2 r^{2}} r^{a}\left[2 \ell^{2} g^{2}(\phi)+\ell^{2} \phi f(\phi)-\frac{(1-a)^{2}}{2} \phi^{2}\right] .
\end{aligned}
$$

Define the local energy and flux by

$$
E(\phi, t)=\int_{0}^{|t|} e(t, r) d r, \quad \operatorname{rmflux}(\phi, t, s)=\int_{|s|}^{|t|}(e-m)(-x, x) x d x,
$$

and integrate (4.12) on the truncated cone $-1 \leq t \leq s<0$ to obtain $-E(\phi, t)+E(\phi, s)+$ $c$ flux $(\phi, t, s)=0$. This implies that

- The energy $E(\phi, t)$ is monotone nonincreasing.

- The flux is bounded by the initial energy, and flux $(\phi, t, s) \rightarrow 0$ as $t \rightarrow 0$.

- Finite energy implies boundedness of solutions (since $e$ has a term of he form $\frac{\phi^{2}}{r^{2}}$ ).

- For solutions $\phi c_{1} \phi^{2} \leq g^{2}(\phi) \leq c_{2} \phi^{2}$ and $|f(\phi)| \leq C|\phi|$. 
- Solutions cannot concentrate on the boundary of the cone i.e.

$$
\phi(-x, x) \rightarrow 0 \quad \text { as } x \rightarrow 0 .
$$

- The annular energy $E_{m}^{\lambda}(\phi, t) \stackrel{\text { def }}{=} \int_{\lambda|t|}^{|t|} e(t, r) r d r \rightarrow 0$ as $t \rightarrow 0$ for $0<\lambda<1$. Moreover by integrating (13) on the truncated cone and letting $s \rightarrow 0$ we obtain:

- For a sufficiently close to 1 we have

$$
2 \ell^{2} g^{2}(\phi)+\ell^{2} \phi f(\phi)-\frac{(1-a)^{2}}{2} \phi^{2} \geq \epsilon \phi^{2},
$$

and this is sufficient to show that $\frac{1}{|t|^{a}} \iint_{K_{t}} \frac{\phi^{2}}{r^{2}} r^{a} d r d t \rightarrow 0$ as $t \rightarrow 0$.

- $E(\phi, t) \rightarrow 0$ as $t \rightarrow 0$ i.e. energy cannot concentrate at $(0,0)$.

To show that small energy implies regularity we make the transformation $\phi=r^{\ell} w$ :

$$
w_{t t}-w_{r r}-\frac{2 \ell+1}{r} w_{r}=\frac{\ell^{2}}{r^{\ell+2}}\left[g(\phi) g^{\prime}(\phi)-\phi\right] .
$$

Since $g(\phi) g^{\prime}(\phi)-\phi=O\left(\phi^{3}\right)$ we have

$$
w_{t t}-w_{r r}-\frac{m-1}{r} w_{r}=c r^{m-4} w^{3} Z(\phi),
$$

where $m=2(\ell+1) \geq 4$ and $Z$ is a smooth function of $\phi$. Since $\phi$ is bounded, we see that $w$ satisfies a wave equation in $m$ space dimensions with nonlinearity of cubic growth. The critical exponent in $m$ dimensions is $2^{*}-1=\frac{m+2}{m-2}=1+\frac{2}{\ell} \leq 3$, but the presence of the $r^{m-4}$ factor makes this essentially a problem with critical exponent.

As in the semilinear wave equation we are going to use the Strichartz estimate (2.9) on $w$ satisfying (4.13):

$$
|w|_{\dot{B}_{q}^{\frac{1}{2}}\left(K_{a}^{b}\right)} \leq C \sqrt{E(w, a)}+c\left|r^{m-4} w^{3} Z(\phi)\right|_{\dot{B}_{q^{\prime}}^{\frac{1}{2}}\left(K_{a}^{b}\right)},
$$

where $q=\frac{2(m+1)}{m-1}$. By Hardy's inequality and Sobolev embedding theorem we estimate the right hand side by:

$$
\left|r^{m-4} w^{3} Z(\phi)\right|_{\dot{B}_{q^{\prime}}^{\frac{1}{2}}\left(K_{a}^{b}\right)} \leq C E(w, a)^{\theta}|w|_{\dot{B}_{q}^{\frac{1}{2}}\left(K_{a}^{b}\right)}^{\gamma},
$$

where $\theta=2 /(m-2)(m-1)$, and hence

$$
|w|_{\dot{B}_{q}^{\frac{1}{2}}\left(K_{a}^{b}\right)} \leq C \sqrt{E(w, a)}+C E(w, a)^{\theta}|w|_{\dot{B}_{q}^{\frac{1}{2}}\left(K_{a}^{b}\right)}^{\gamma},
$$

with $\gamma>1$. Since $E(w, a) \leq c E(\phi, a)$ and we have shown $E(\phi, a)<\epsilon$ for $a$ close enough to 0 , this implies that $|w|_{\dot{B}_{q^{\prime}}^{\frac{1}{2}}\left(K_{a}^{b}\right)}$ is bounded.

Higher regularity for $w$ can now be obtained by differentiating the equation and repeating the above argument. Eventually we gain enough regularity to deduce $w \in L^{\infty}$. Higher energy estimates can then be used to improve this to $w \in C^{\infty}$, or as regular as the data. This completes the proof of regularity for $\phi$.

4.4. Singularities and non-uniqueness. Since equations (4.1) are invariant under scaling $u_{\lambda}(x, t) \stackrel{\text { def }}{=} u(x / \lambda, t / \lambda)$, then singularities can develop by simply forcing the solution 
to concentrate at a point. Therefore we look for solutions that are self similar

$$
u=v(x / t) .
$$

These solutions are constant on rays emanating from the origin, thus possibly leading to a derivative singularity at the origin. Substituting this ansatz into equation (4.1) we obtain the equation of equivariant harmonic maps from the hyperbolic space $\mathbb{H}^{n}$ into $N$. This is to be expected since if we use on space time the coordinate system

$$
\sigma=\sqrt{t^{2}-r^{2}}, \quad \rho=r /|t|
$$

the hypersurface $\sigma=1$ is the hyperbolic space $\mathbb{H}^{n}$, and solutions that are independent of $\sigma$ are harmonic maps from the hyperbolic space $(\sigma=1)$ into $N$. If such maps exist and are regular (including regularity at infinity), then we can show that the Cauchy problem with regular initial data can develop singularities in finite time. This is accomplished by using these maps as initial data at $t=-1$ and noting that the solution

$$
u=v\left(\frac{x}{-t}\right)
$$

develops a singularity in the derivative as $t \rightarrow 0$.

For equation (4.1) to have an equivariant self-similar solution $\phi(r /-t), \phi$ has to satisfy the following ODE on the interval $[0,1]$ :

$$
\phi^{\prime \prime}+\left(\frac{n-1}{r}+\frac{(n-3) r}{1-r^{2}}\right) \phi^{\prime}=\frac{k}{r^{2}\left(1-r^{2}\right)} f(\phi),
$$

where $f:=g g^{\prime}$ and $k:=\ell(\ell+n-2), \ell \in \mathbb{N}$.

The solution $\phi$ has to vanish at $r=0$ and has to be smooth on $[0,1]$. We will establish the existence of such solutions for odd space dimensions $n$, provided the metric on the target $N$ satisfies certain equations, which we will refer to as $n$-boundary conditions. These equations can be derived by requiring a solution to (4.15) to be smooth at $r=1$. For example for $n=5$ if we plug in a smooth function $\phi(r)=a_{0}+a_{1}(v-1)+\ldots$ into equation (4.15) we obtain

$$
2 a_{1}=k f\left(a_{0}\right), \quad-2 a_{1}=k f^{\prime}\left(a_{0}\right) a_{1} .
$$

Thus we obtain the boundary conditions

$$
f\left(a_{0}\right)\left(2+k f^{\prime}\left(a_{0}\right)\right)=0 .
$$

Since $k=\ell(\ell+n-2)$ and $\ell$ is an arbitrary integer then the above eqution has a solution if $\exists a_{0}>0$ such that either $f\left(a_{0}\right)=0$ or $f^{\prime}\left(a_{0}\right)<0$ (since $f^{\prime}(0)>0$ ). Similar calculations lead to an equation for $a_{0}=\phi(1)$ in all odd dimensions. The $n$-boundary conditions are polynomial equations in $f\left(a_{0}\right), f^{\prime}\left(a_{0}\right), f^{\prime \prime}\left(a_{0}\right), \ldots, f^{(m)}\left(a_{0}\right)$ where $m=(n-3) / 2$. These equations are always satisfied if $\exists a_{0}>0$ such that $f\left(a_{0}\right)=0$, i.e. the $\operatorname{target} N$ is not geodesically convex. These conditions allow us to prove the following

LEMMA 4.1. If a solution of (4.15) satisfies the boundary condition and $\phi \in C^{\frac{1}{2}}[0,1]$, then $\phi$ is smooth at $r=1$.

This lemma can be proved by integrating the ODE close to $r=1$ and using a bootstrap argument [3]. To show that the ODE has a smooth solution we distinguish two cases. 
Non-convex case. Assume there exists a $\phi_{*}>0$ such that the metric on $N$ satisfies $g^{\prime}\left(\phi_{*}\right)=0$ and $g^{\prime \prime}\left(\phi_{*}\right)<0$. In this case the function $f$ has a second zero. Smooth solutions of the ODE can be found by minimizing the functional

$$
E[\phi]:=\frac{1}{2} \int_{0}^{1}\left\{\phi^{\prime}(r)^{2}+\ell(\ell+n-2) \frac{g^{2}(\phi)-g^{2}\left(\phi_{*}\right)}{r^{2}\left(1-r^{2}\right)}\right\} \frac{r^{n-1} d r}{\left(1-r^{2}\right)^{(n-3) / 2}} .
$$

subject to the condition $\phi(1)=\phi_{*}$. Any minimizing sequence $\left\{\phi_{n}\right\}$ for $E$ is bounded and therefore has a weakly convergent subsequence $\phi_{n} \stackrel{w}{\rightarrow} \bar{\phi}$ in $H^{1}$. Moreover for $\ell$ large enough we can exhibit a function $\tilde{\phi}=\phi_{*}+\varepsilon \eta_{\varepsilon}$, with

$$
\eta_{\epsilon}(r):=\left\{\begin{array}{l}
\left(r^{-\beta}-1\right)^{\alpha}, \epsilon \leq r \leq 1, \\
\left(\epsilon^{-\beta}-1\right)^{\alpha}, 0 \leq r \leq \epsilon,
\end{array} \quad \text { where } \alpha:=\frac{n+1}{4}, \beta:=2 \frac{n-2}{n+1} .\right.
$$

such that $E(\tilde{\phi})<0$. By lower semi continuity of $E$ we have $E(\bar{\phi})<0$ and $\bar{\phi} \neq \phi_{*}$ is a minimizer. Since $\bar{\phi}$ solves the ODE (4.15), then $\bar{\phi}$ is smooth on the open interval $(0,1)$. Heuristically, for $r \sim 0$ solutions of the ODE behave like $\phi \sim c_{1} r^{\ell}+c_{2} r^{-(\ell+n-2)}$. Since $\bar{\phi}$ has finite energy then $\bar{\phi} \sim c_{1} r^{\ell}$ for $r \sim 0$ and $\bar{\phi}$ is smooth on [0,1). To show that $\bar{\phi}$ is smooth at $r=1$, note that finite energy implies $\bar{\phi} \in C^{\frac{1}{2}}([0,1])$, and that $\bar{\phi}(1)=\phi_{*}$ satisfies the $n$-boundary condition. By Lemma $4.1, \bar{\phi} \in C^{\infty}([0,1])$.

Convex case. In this case we have $g^{\prime} \neq 0$, and the nonlinear term in the ODE satisfies $\phi f(\phi)>0$ if $\phi \neq 0$. To find a smooth solution in this situation we use a shooting argument. First we solve the initial value problem $\phi_{c}(r)=c r^{\ell}$ for $r$ close to zero and then show that we can choose $c$ so that $\phi_{c}(1)$ satisfies the $n$-boundary condition. The steps in the proof proceed as follows:

- $\forall c \in[0, \infty) \exists ! \phi_{c} \in C^{\frac{1}{2}}([0,1])$ solution to $(4.15)$.

- The map $c \longrightarrow \phi_{c}(1)$ is continuous from $[0, \infty) \rightarrow[0, \infty)$.

- Given $\tilde{f}<f$ such that $\tilde{f}^{\prime}>0$, then $\tilde{\phi}_{c}<\phi_{c}$ where $\tilde{\phi}_{c}$ is a solution to the ODE with $f$ replaced by $\tilde{f}$.

- $\lim _{c \rightarrow \infty} \tilde{\phi}_{c}(1) \longrightarrow+\infty$.

Since $\phi_{0}(1)=0$ the above implies that the range of $\phi_{c}(1)$ is $[0, \infty)$. Therefore we can choose $c^{*}$ so that $\phi_{c^{*}}(1)$ satisfies the $n$-boundary condition. By Lemma $4.1, \phi_{c^{*}}$ is smooth on $[0,1]$. Using these smooth solutions as initial data for (4.1) we have:

THEOREM 4.5. Let $N$ be a rotationally symmetric manifold and let $n \geq 3$ be odd. If the metric on $N$ satisfies the $n$-boundary condition, then there exist smooth initial data such that the corresponding Cauchy problem (4.1) develops singularities in finite time.

Moreover for the non-convex case we can construct non-unique solutions in the following manner: Solutions to the ODE (4.15) have an asymptotic behaviour as $r \rightarrow \infty$

$$
\phi(r)=a+\frac{b}{r}+O\left(\frac{1}{r^{2}}\right) .
$$

This implies that for the Cauchy data

$$
\phi(0)=a, \quad \partial_{t} \phi(0)=-\frac{b}{r},
$$


equation (4.6) has $\phi(r / t)$ as a weak solution in $H_{\text {loc }}^{s}\left(\mathbb{R}^{n}\right) \forall s<n / 2$. However since $\phi_{*}$ is also a solution to the ODE one can check that

$$
\bar{\phi}(t, r)= \begin{cases}\phi\left(\frac{r}{t}\right), r>t, \\ \phi_{*}, \quad r<t,\end{cases}
$$

is also a weak solution corresponding to the same data.

THEOREM 4.6. Let $N$ be rotationally symmetric manifold and let $n \geq 3$ be odd. If $N$ is not geodesically convex, then there exist initial data which belong to $H^{s}, \forall s<\frac{n}{2}$, such that the Cauchy problem (4.1) has non-unique solutions.

For even space dimensions we cannot prove the existence of smooth initial data where the corresponding solution to the Cauchy problem (4.1) is self-similar. Of course we can generate singular solutions in even dimensions by considering self-similar solutions $u(\omega, r / t)$ in odd space dimensions $\mathbb{R}^{n+1}$ as solutions on $\mathbb{R}^{n+2}$ which are independent of the $x^{n+1}$ variable. However such a solution will not lead to optimal ill-posedness results since singularities will develop on the whole $x^{n+1}$ axis, and the solution $u \notin H^{s}\left(\mathbb{R}^{n+1}\right) \forall s<$ $(n+1) / 2$ as $t \rightarrow 0$. However this problem can be rectified by composing the self-similar solution on $\mathbb{R}^{n+1}$ with the Lorentz boost in the $x^{n+1}$ direction on $\mathbb{R}^{n+2}$

$$
L\left(t, r, \omega, x^{n+1}\right)=\left(\frac{\beta t+x^{n+1}}{\sqrt{\beta^{2}-1}}, r, \omega, \frac{t+\beta x^{n+1}}{\sqrt{\beta^{2}-1}}\right),
$$

to produce a singular traveling wave on $\mathbb{R}^{n+2}$

$$
\bar{u}\left(t, r, \omega, x^{n+1}\right)=u\left(\omega, \frac{\sqrt{1-\beta^{2}} r}{\beta t+x^{n+1}}\right) .
$$

This traveling wave propagates with a speed $\beta>1$ and at any instant of time is singular at a point $r=0, x^{n+1}=-\beta t$. Therefore if we consider initial data equal to the traveling wave in a ball of radius $(\beta+1) / 2$, then the solution corresponding to such initial data will develop a singularity at $t=-\frac{1}{2}$. Moreover $\forall s<(n+1) / 2 \bar{u} \in H_{\text {loc }}^{s}$ as $t \rightarrow-\frac{1}{2}$.

TheOREm 4.7. Let $N$ be rotationally symmetric manifold and let $n \geq 3$ be odd. Then

1. If the metric on $N$ satisfies the $n$-boundary condition then there is a singular traveling wave map from $\mathbb{R}^{n+2}$ into $N$.

2. If $N$ is not geodesically convex then there exist initial data in $H^{s}\left(\mathbb{R}^{n+1}\right), \forall s<$ $(n+1) / 2$, such that the corresponding Cauchy problem (4.1) has non-unique solutions.

\section{References}

[1] J. Bergh and J. Löfström, Interpolation Spaces, Grundlehren 223, Springer-Verlag, 1976.

[2] P. Brenner and W. von Wahl, Global classical solutions of nonlinear wave equations, Math. Z., 176:87-121, 1981.

[3] T. Cazenave, J. Shatah, and A. Tahvildar-Zadeh, Harmonic maps of the hyperbolic space and development of singularities for wave maps and Yang-Mills fields, preprint, 1995. 
[4] J. Ginibre and G. Velo, The Cauchy problem for the $O(N), \mathbb{C} P(N-1)$ and $G \mathbb{C}(N, P)$ models, Ann. Physics, 142:393-415, 1982.

[5] J. Ginibre and G. Velo, The global Cauchy problem for the nonlinear Klein-Gordon equation, Math. Z., 189:487-505, 1985.

[6] J. Ginibre and G. Velo, The global Cauchy problem for the nonlinear Klein-Gordon equation revised, Ann. Inst. H. Poincaré, Analyse Non Linéaire, 6:15-35, 1989.

[7] M. Grillakis, Regularity and asymptotic behavior of the wave equation with a critical nonlinearity, Ann. of Math., 132:485-509, 1990.

[8] M. Grillakis, Classical solutions for the equivariant wave map in 1+2-dimensions, preprint, 1991.

[9] M. Grillakis, A priori estimates and regularity for nonlinear waves, Communication at ICM 94, Zurich, 1994.

[10] H. Triebel, Interpolation Theory, Function Spaces, Differential Operators, Math. Lib., volume 18. North-Holland, 1978.

[11] K. Jörgens, Das Anfangswertproblem im Grossen für eine Klasse nichtlinearer Wellengleichungen, Math. Zeit., 77:295-308, 1961.

[12] L. Kapitanski, Global and unique weak solutions of nonlinear wave equations, Math. Res. Lett., 1:211-223, 1994.

[13] S. Klainerman and M. Machedon, Smoothing estimates for null forms and applications, Duke Math. Jour., 81:99-133, 1995.

[14] H. Lindblad and C. Sogge, On existence and scattering with minimal regularity for semilinear wave equations, J. Func. Anal., 130:357-426, 1995.

[15] B. Marshall, W. Strauss, and S. Wainger, $L^{p}-L^{q}$ estimates for the Klein-Gordon equation, J. Math. Pures et Appl., 59:417-440, 1980.

[16] H. Pecher, $L^{p}$-Abschätzungen und klassische Lösungen für nichtlineare Wellegleichungen, I, Math. Z., 150:151-183, 1976.

[17] G. Ponce and T. Sideris, Local regularity of nonlinear wave equations in threee space dimensions, Comm. Partial Diff. Eq., 18:169-177, 1993.

[18] J. Rauch, I. The $u^{5}$ Klein-Gordon equation. II. Anomalous singularities for semilinear wave equations, in H. Brézis and J. L. Lions, editors, Nonlinear Partial Differential Equations and Their Applications, volume 53, pages 335-364. Pitman, 1981.

[19] I. Segal, Nonlinear Semi Groups, Ann. Math., 78:339-364, 1963.

[20] J. Shatah and M. Struwe, Regularity results for nonlinear wave equations, Ann. Math., 138:503-518, 1993.

[21] J. Shatah and M. Struwe, Well-posedness in the energy space for semilinear wave equations with critical growth, IMRN, 7:303-309, 1994.

[22] J. Shatah and A. Tahvildar-Zadeh, Regularity of harmonic maps from the Minkowski space into rotationally symmetric manifolds, Comm. Pure Appl. Math., XLV:947-971, 1992.

[23] J. Shatah and A. Tahvildar-Zadeh, On the Cauchy problem for equivariant wave maps, Comm. Pure Appl. Math, 47:719-754, 1994.

[24] W. Strauss, Nonlinear invariant wave equations, in G. Velo and A. S. Wightman, editors, Invariant Wave Equations, pages 197-249. Springer-Verlag, Berlin, 1978.

[25] W. Strauss, Nonlinear Scattering Theory at Low Energy, J. Funct. Anal., 41:110-133, 1981.

[26] R. S.Strichartz, Restrictions of Fourier transforms to quadratic surfaces and decay of solutions of wave equations, Duke Math. J., 44:705-714, 1977. 
[27] M. Struwe, Globally regular solutions to the $u^{5}$ Klein-Gordon equation, Annali Sc. Norm. Sup. Pisa (Ser. 4), 15:495-513, 1988.

[28] M. Struwe, Geometric Evolution Problems, Park City Geom. Ser. of the Amer. Math. Soc., Providence, R.I., 1992. 